\title{
On the Market Value of Information Commodities. I. The Nature of Information and Information Commodities
}

\author{
Abbe Mowshowitz* \\ Rotterdam School of Management, Erasmus University-Rotterdam, 3000 DR Rotterdam, The Netherlands
}

This article lays the conceptual foundations for the study of the market value of information commodities. The terms "information" and "commodity" are given precise definitions in order to characterize "information commodity," and thus to provide a sound basis for examining questions of pricing. Information is used by marketplace actors to make decisions or to control processes. Thus, we define information as the ability of a goal-seeking system to decide or control. By "decide" we mean choosing one alternative among several that may be executed in pursuit of a well-defined objective. "Control" means the ordering of actions. Two factors make it possible to turn something into a commodity: (1) appropriability, and (2) valuability. If something cannot be appropriated (i.e., owned), it cannot be traded; moreover, if it cannot be valued, there is no way to determine for what it might be exchanged. We define an information commodity as a commodity whose function it is to enable the user, a goal-seeking system, to obtain information, i.e., to otain the ability to decide or control. Books, databases, computer programs, and advisory services are common examples of information commodities. Their market value derives from their capacity to furnish Information.

\section{Introduction}

This is the first in a series of three articles on the market value of information, or more precisely, information commodities. The market for information commodities, like other markets, may be analyzed in terms of supply and demand. Market prices reflect some sort of equilibrium (however imperfect) between these two forces. Our aim in this and the following articles (Mowshowitz, 1992a, b) is to elaborate methodologies for estimating lower and upper bounds on the market price for information commodities. By lower bound, we mean the lowest price (relative to a particular production method) the producer can charge without incurring losses; by upper bound, we mean the highest price (rela-

\footnotetext{
*Author's permanent affiliation: Department of Computer Science, The City College of the City University of New York.

Received March 7, 1991; revised May 20, 1991; accepted May 29,

(1) 1992 by John Wiley \& Sons, Inc.
} 1991. tive to a particular application) the consumer can pay without sacrificing profits.

Determining lower bounds requires an analysis of the supply side or the producer's perspective; estimating upper bounds calls for analyzing the demand side or the user's perspective. Note that we are concerned primarily with information commodities that have a derived demand. We do not attempt to determine the market value of information commodities produced for final consumption by consumers. Rather, the focus is on evaluating information commodities which are used to produce something else, either another information commodity or a conventional product or service.

Before we can investigate price determination, a number of fundamental issues must be clarified. In particular, we must explain what precisely we mean by "information commodity." To do this, we must first define the terms "information" and "commodity." With serviceable definitions of these basic concepts, we will be in a position to characterize "information commodities" and to study their pricing.

The discussion of fundamental issues is essential for both the supply and demand perspectives, and thus it is presented as an independent article.

\section{Information}

Information has many faces. It appears as pure knowledge coveted by scholars, as skill acquired by children, as a requisite for individual or organizational decision making, as the stuff pumped through communication or control channels, as the specification of a product or production process, etc. Whether or not these manifold forms of information are different aspects of a unity constitutes a fascinating and significant philosophical question. Fortunately, we do not have to resolve this question here.

We are concerned with the economics of information. ${ }^{1}$ In particular, we want to know how certain kinds of information acquire value in the marketplace, and how that value is reflected in the equilibrium price for

\footnotetext{
${ }^{1}$ For an overview of information economics and extensive bibliographies, see Lamberton (1984a, b).
} 
those kinds of information. This narrow focus excludes considerations of value other than those germane to exchange relations in the marketplace. The value that a mathematician places on a solution to an open problem in mathematics is not primarily economic value. Nor is it economic value that a reader attributes to the meaning of a novel. Clearly, information is valued in many different ways. Thus we make explicit our restriction of the term "value of information" to the economics of the marketplace.

Marketplace actors use information to make decisions and to control processes. The contexts of use are exchange, production, and consumption. Exchange and production use information as an instrumental or intermediate element; but information may also be the object of final consumption.

Information is used by sellers in exchange transactions to determine the best price to charge for some item; it is used by buyers to determine the best price to pay.

In production, information is used to make a variety of decisions about acquisition, allocation, use, and distribution of resources. It is also used to control production processes and to specify the composition or production method of parts and processes.

Information is an object of final consumption in many different contexts. For example, one pays to take courses in part because education enables one to qualify for a job; but some courses have only the most tenuous connection to career development. One sometimes reads instructional books for pleasure or personal growth. Some people even read financial magazines just out of (nonfinancial) interest in the stock market. These uses of information are no more intermediate than is the consumption of bread. If reading for personal growth is seen as instrumental to one's functioning as, say an engineer, then so is the consumption of bread instrumental, for bread provides sustenance for the body supporting the job-related activities of the engineer.

The difference between intermediate and final consumption of information is the same as the analogous distinction in the case of tangible economic goods. For intermediate goods it is possible to estimate both the vendor's minimum asking price and the buyer's maximum offering price on the basis of exchange relations. On the other hand, an item destined for final consumption does not play a role in further production, so the buyer cannot determine the maximum, justifiable price on this basis. The vendor of an intermediate good must pass on the production costs to the buyer; the buyer of such a good can only justify a purchase if the use of the item purchased yields a return at least equal to the purchase price. The buyer of an item destined for final consumption has no intention of using it to produce something else to be offered for sale. ${ }^{2}$

\footnotetext{
${ }^{2}$ See King (1982) for discussion of information products and services marketed to end users.
}

The pricing model we intend to introduce applies only to the production of information for sale, and the use of information as an intermediate economic good. However, the definition of information commodity, toward which we are aiming, applies to information in all its economic contexts.

Many definitions of information have been proposed. The most prominent approach, linking information to probability distributions, was elaborated by Shannon (1949). This approach is distinguished by its introduction of a quantitative measure. Shannon's celebrated entropy measure, because of its extraordinary usefulness in communications engineering, has proven irresistable to observers in fields as divergent as biology and music. ${ }^{3}$ But it is generally agreed that Shannon's measure, which equates information with uncertainty, is limited in its applicability. ${ }^{4}$

In any event, it should be noted that a measure is not the same as a definition. A measure presupposes a definition which specifies the domain of the measure's applicability. The definitions of information thus far proposed fail to specify the domain. We remedy this deficiency by viewing information as a property of certain kinds of systems.

Information is used by marketplace actors to make decisions or to control processes. This observation leads us to propose the following definition: Information is the ability of a goal-seeking system to decide or control. ${ }^{5}$ By "decide" we mean choosing one alternative among several that may be executed in pursuit of a welldefined objective. "Control" means the ordering of actions that may be undertaken to achieve a well-defined objective. A goal-seeking system is one whose actions are designed to achieve a particular objective. Notc that choosing and ordering are planning-related. Neither implies the cxecution of planned actions.

This definition implicitly distinguishes between choice and order, on the one hand, and the realization of choices and ordered sets of actions, on the other hand. Thus, a goal-seeking system may be able to execute actions but, in the absence of information, will not be able to make choices or to control processes. A computer without a FORTRAN compiler, for example, has the capability of executing programs in general, but lacks the ability to translate a FORTRAN program into exe-

\footnotetext{
${ }^{3}$ For further discussion of the many applications of Shannon's information theory, see Mowshowitz (1987).

${ }^{4}$ Hintikka $(1984$, p. 175) demonstrates this limitation by tracing the consequences of "clues ... to the logical and mathematical behavior of the concept of information." Hoffman (1980, p. 293) proposes to overcome the limitation of the uncertainty measure by defining information as "an aggregate... of statements, of facts and/or figures which are conceptually... interrelated."

${ }^{5}$ Compare this definition with the classical view. For example: (1) "Information ... consists of events tending to change [individuals' subjective] probability distributions [over possible states of the world]" Hirshleifer, 1973). (2) "Intuitively, a change in information is a change in the probability distribution of states of the world" (Arrow, 1979)
} 
cutable code because it does not have the ordered set of instructions that constitutes a FORTRAN compiler.

Our definition of information as the ability to decide or control is consistent with Shannon's interpretation of information as the removal of uncertainty. However, the economic value of $\mathrm{H}$ bits of information depends on the expected payoff associated with the use of those $\mathrm{H}$ bits. Two messages from different ensembles may have exactly the same entropy or uncertainty, but differ markedly in the respective payoffs expected from their use. Shannon's entropy measure may be used to characterize the uncertainty removed by the receipt of a message in a decision-making context. But that measure tells us nothing about the economic value of a message to the recipient/decision maker. ${ }^{6}$

The relationship between uncertainty and the economic value of information is analogous to the relationship between quantity and the economic value of tangible goods. Uncertainty or entropy measures the "amount of information" just as volume or weight measures the "amount of tangible goods." But information, like tangible goods, occurs in many different varieties. An ounce of butter does not have the same economic value as an ounce of gold. Similarly, the specification of a commercial baking process and grandmother's recipe for apple pie are likely to diverge in economic value even if they happen to measure the same on the uncertainty scale. The world of information, like that of tangible goods, is highly differentiated and encompasses things of divergent economic value.

The foregoing analysis agrees with Marschak (1959) on the relation between cost and amount (as measured by entropy or uncertainty) of information. Marschak (1959, p. 81) argucs that "[amount of information] is not identical with the value of information ... shown to determine the demand price. But it is presumably related to cost, and hence the supply price, of information."

Shannon's entropy function was developed to measure the carrying capacity of information channels. Failure to realize that information is not an undifferentiated essence has led to many inappropriate adaptations of this measure outside the field of communications engineering. Entropy (as channel capacity) is useful for measuring the maximum amount of information (of any kind whatsoever) that can be pumped through a medium like a pair of copper wires; analogously, volume (as carrying capacity) is useful for measuring the maximum amount of fluid (of whatever kind) that can be carried from port to port in a vessel such as a tanker. Measures of uncertainty and volume are extremely useful for carriers of information and fluids, respectively; but they give virtually no clue as to the economic value to the user of what is being carried.

The way in which information is priced in the marketplace appears at first glance to be radically different

\footnotetext{
${ }^{6}$ For further discussion of the relation between uncertainty and meaning see Langlois (1982) and Mowshowitz (1987).
}

from the way energy is priced. Energy is priced according to quantity measures such as number of BTUs. A quantity of oil yielding a given number of BTUs will cost the same no matter what use is made of it. What about information? As we explained earlier, the economic value of information to a user cannot be determined solely by the amount of uncertainty-reduction it promises. What the comparison with energy reveals is that there are radical differences in the costs of producing different types of information with the same uncertainty-reduction value. The resulting variations in producer prices, coupled with variations in potential buyers' willingness to pay, yields different rates for the same amount of information.

Information, defined as "ability," rather than "stuff," has two complementary aspects: (1) the ability to observe or experiment, and (2) the ability to express beliefs. ${ }^{7}$ Consider the following observation about the water in a lake: "The current temperature of the water is $c$ degrees Celsius." Assuming temperature to be the only consideration, this observation would enable one to decide whether or not to go swimming. But the observation (or fact) giving the current temperature is not the only element in the decision process. The ability to decide hinges upon a conditional statement and an inference rule, which are typically implicit in the decision process.

In our example, the conditional is of the form: "If the water temperature is between $a$ and $b$ degrees Celsius, then one can swim comfortably." Now, assuming the value of $c$ is between $a$ and $b$, one could infer, by modus ponens, the statement "one can swim comfortably." Note that the conditional statement together with the modus ponens inference rulc would not, by themselves, be sufficient to make a decision about swimming. The condition expressed in the antecedent must be ascertained, i.e., the water temperature must be measured. To accomplish this, a measurement procedure must be available.

This example suggests interpreting information, i.e., the ability to decide or control, as a system consisting of two interacting components. The first of these might be called the belief subsystem; the second, the command subsystem. The belief subsystem encompasses declarative statements and inference rules; the command subsystem is composed of procedural statements together with rules for generating such statements. ${ }^{8}$ Note that the system characterized by these two components does not have any effector mechanisms. That is to say, it does not itself draw inferences or make observations. Such actions are performed by independent agents.

\footnotetext{
${ }^{7}$ The significance of this complementarity was brought to the author's attention by Professor Giovanni Criscuolo.

"The terms "declarative" and "procedural" are used here in a way that is entirely analogous to their use in the area of knowledge representation. See Winograd (1975) for a discussion of the relationship between declarative and procedural forms of knowledge representation.
} 
The information-ability to decide or control-represented by the system sketched above lies in the relationship between belief and command (or declaration and procedure). So-called facts, like the water temperature in the example, confer the ability to decide when the relevant conditionals are in the belief system. Conversely, statements of belief confer such ability when the requisite observation procedures are in the command system. In short, the key to the ability that constitutes information lies in the complementarity of declarative and procedural statements.

The declarative statements of the belief subsystem may be modeled as statements in predicate logic. These include descriptions of reality and arbitrary boolean combinations of descriptive statements. The statement about water temperature, discussed in the above example, illustrates a description of reality. Here are some more examples:

(1) "The sky is overcast with dark clouds."

(2) "The temperature of the mixture in the blue container is 120 degrees Celsius."

(3) "John exceeded his sales quota this month."

(4) "Companies $X, Y$, and $Z$ have all placed orders in excess of $\$ 1000$ over the past quarter."

As explained earlier, statements such as these, together with conditionals, facilitate decision making. Assuming one would prefer to avoid getting soaked in the rain, knowledge of current weather conditions as given in statement (1) enables one to decide whether or not to wear rain gear. Knowing the current temperature of a mixture as given in (2) allows one to decide whether or not to open a valve or to add another ingredient to the mixture.

All of the above are examples of statements in propositional logic, i.e., they are either true or false. To accommodate the full range of descriptive statements, we need to go beyond propositional logic to predicate logic. We should like for example to admit statements of the form "Every product whose number begins with the string 'acd2' has been discontinued." With this extension to quantified statements, we have an adequate characterization of declarative statements.

One further example may help to clarify the range of possibilities included in this characterization. Information obtained by searching a database typically consists of a set of items. Suppose such a set $S$ consists of elements $a, b, c, d$, and $e$ which satisfy the criteria that defined the search. To represent the set as a proposition, we first define the predicate $A$ to mean "satisfying the search criteria." Then the set $S$ is the statement:

$$
A(a) \wedge A(b) \wedge A(c) \wedge A(d) \wedge A(e)
$$

Predicate logic suffices for declarative statements, but we need something more. Recipes, procedures, algorithms, and the like involve statements of the form "Add two teaspoons of sugar." or "Open the pressure valve if the sensor gives a reading above 20 psi." or
"Execute statement number 30 if $x-y$ is less than zero." These are all imperative statements, calling for action, and do not have a truth value. Thus, procedures, algorithms, process specifications, etc. comprise a variety of statement that is different from the declarative type. These are what we have been calling procedural statements. In the belief-command characterization of information as an ability, procedural statements prescribe how to obtain descriptions of reality.

In summary, declarative statements express belief about the state of the world; procedural statements prescribe how to transform the world. The following recursive definitions summarize the foregoing discussion of these two types of statements.

Declarative Statements (DS).

(Ia) Any statement in first-order logic is DS.

(IIa) If $\mathrm{R}$ and $\mathrm{S}$ are $\mathrm{DS}$, then $\mathrm{R}{ }^{*} \mathrm{~S}$ is also DS, where $*$ is any boolean operation.

Procedural Statements (PS).

(Ib) Any executable imperative statement is PS. (Executable means that the action or injunction of the imperative can be realized. Realizability is dependent on the universe of discourse. For example, the imperative "Go to Hell" would not be PS for an atheist.)

(IIb) If I and J are PS, then IJ and JI are PS, where $\mathrm{XY}$ signifies $\mathrm{X}$ followed by $\mathrm{Y}$.

An economically important attribute of statements (both declarative and procedural) is the extent to which they can be described by partial extracts or indexes. A statement's extractability or indexability gives some insight into exchange possibilities in the marketplace. This follows from the fact that the less a statement can be indexed, the more the statement itself must be used to represent its usefulness to a potential purchaser. In the limiting case, usefulness cannot be ascertained without examining the item in its entirety; in other words, there is no way to index the statement apart from itself.

Suppose, for example, a report saying "War has just broken out between countries $A$ and $B$ in region $X$ " is issued by an exclusive and highly reliable source in that region. Suppose further that region $X$ is isolated from the rest of the world and that the existence or nonexistence of a state of war between countries $A$ and $B$ has material implications for production or distribution of some commodity, say oil. From outside the region there is no way to estimate the value of the information without having it. That is to say, nothing short of knowing that a state of war exists will enable participants in the oil market to profit from the information contained in the report.

A precise definition of indexability would be extremely useful, but, like many useful things, it is not easy to obtain. Indeed, it may not be possible to produce a definition that applies to all universes of discourse. 


\section{Commodities}

Armed with a definition of "information" we need only clarify the meaning of "commodity" to arrive at a precise definition of "information commodity." Economists usually treat the term "commodity" as a primitive requiring no definition. Unfortunately, this approach fosters considerable ambiguity, since the word "commodity" means different things to different users. Absent help from the specialists, we resort to catalogues of common usage.

The Oxford English Dictionary defines commodity in the context of commerce as "a kind of thing produccd for use or sale, an article of commerce, an object of trade." Other dictionaries give similar definitions: "an article of trade or commerce that can be transported, especially an agricultural or mining product" (American Heritage Dictionary); "an economic good ... a product of agriculture or mining ... an article of commerce especially when delivered for shipment" (Webster's Ninth New Collegiate Dictionary).

Except for the qualifying phrases "can be transported" and "product of agriculture or mining" the wording used in these three definitions is the same. To get unanimity, one might argue that transportability is implicit in the OED's phrase "produced for sale or use." This would seem to exclude (naturally occurring) land and some other nontransportable things, and thus to be more or less in accord with the other definitions. The second qualifier-product of agriculture or miningsimply indicates the most commonly mentioned varieties of commodity. Intangible things such as patents, copyrights, and other forms of intellectual property seem to be excluded from consideration altogether.

Unfortunately, the dictionary definitions fail to specify precisely what is meant by an article of trade. Business people of ten use the word commodity to refer to relatively high-volume products, as distinguished from specialized or one-of-a-kind articles. So, a specialized machine built for a specific client would not be a commodity, whereas a standardized drill press would be. This distinction introduces replicability as an attribute of commodity. Since the specialized machine may very well be as transportable as the standardized one, replicability is not the same as transportability. Are these attributes essential to the notion of commodity? We think not.

Two factors make it possible to turn things into objects of trade: (1) appropriability, and (2) valuability. If something cannot be appropriated (i.e., owned), it cannot be traded; moreover, if it cannot be valued, there is no way to determine for what it might be exchanged. Appropriation and valuation are both social processes. The former implies a legal system that defines rights in property and the conditions under which property can be transferred; the latter implies collective knowlcdge of the uses of things in relation to human needs and wants.
A simple mathematical representation may help to clarify this interpretation of a commodity. Let $\mathbf{G}$ be a set of economic goods, where the notion of economic goods (e.g., land, natural resources, farm products, manufactured goods, labor, intellectual property, financial instruments, etc.) is a primitive in the model; and let $\mathbf{O}$ be a set of ownership units. Note that the elements of $\boldsymbol{O}$ depend on the legal system. In modern America, individuals, partnerships, corporations, jointtenants, tenants-in-common, and perhaps other entities would qualify as ownership units.

Now we define appropriability as a function A mapping $\mathbf{G}$ into $\mathbf{O}$ as follows:

$$
A(g)=\left\{\begin{array}{l}
u, \text { if } u \text { owns } g \\
\text { undefined otherwise }
\end{array}\right.
$$

The inclusion of various types of owning units in $\mathbf{O}$ allows us to handle the case of shared ownership. If, for example, several individuals own something jointly as shareholders or partners, the owning unit of which they are members would be an element of the set $\mathbf{O}$. Thus, if an economic good in $\mathbf{G}$ is assigned an owner in $\mathbf{O}$, that owner is unique.

Valuation may be defined as a function mapping equivalence classes of economic goods into the rcal numbers. An equivalence class of economic goods is a set of individual items or tokens of a given type (e.g., all toasters with model number 783425 produced by United Widgets, Inc.). Two tokens of the same type are "essentially the same," meaning that neither producer nor user would be able to distinguish one from another.

The precise meaning of this indistinguishability varies from one market context to another. For example, unlike discrete products such as toasters and automobiles, bulk goods like wheat and petroleum are typically traded in multiples of volume units determined by their respective markets. So, equivalence for wheat would mean the interchangeability of two bushels of the same type, grade, etc.

Whereas ownership involves tokens, valuation applies to equivalence classes of tokens. The valuation function $\mathbf{V}$ maps the equivalence classes of $\mathbf{G}$ into $\mathbf{R}$, the set of real numbers:

$$
\mathbf{V}([g])=\left\{\begin{array}{c}
c, \text { if } c \text { is the nonzero average } \\
\text { price paid for any token in }[g] \\
\text { undefined otherwise }
\end{array}\right.
$$

Since the market prices of tokens belonging to the same type may vary, we choose the average in order to assign a unique number to a given type. The entire set of reals (rather than the nonnegative reals) is used in the definition, because some equivalence classes of economic goods (such as toxic byproducts of industrial processes) may have negative values. Since some byproducts are owned and incur costs in disposal, they must be treated as equivalence classes with negative value. 
Having specified the meanings of ownership and valuation, we are in a position to define commodity. A commodity is an equivalence class $[g]$ of economic goods such that $\mathbf{V}([g])=c$ (nonzero real number) and $\mathbf{A}(x)=u$ for every $x$ in $[g]$. The two conditions specify, respectively, that $[g]$ has a unique value, and every member of $[g]$ has a unique owner. Note that $[g]$ could consist of a single element, i.e., $[g]=\{e\}$, so we allow unique goods such as artistic works within our definition of commodity. If a work of art has an owner and a market value, it is a commodity.

This definition of commodity introduces two sets, $\mathbf{G}$ and $\mathbf{O}$, and two functions, $\mathbf{A}$ and $\mathbf{V}$. Both the sets and the functions may vary over time-economic goods and owners have finite life spans; ownership is transferrable and prices vary with changes in the marketplace. Thus, we may indicate the dependence of $\mathbf{G}, \mathbf{O}, \mathbf{A}, \mathbf{V}$ on time by the use of a subscript $t$ representing time. The values assumed by $t$ will depend on the context. When the time period is specified and fixed by context, we can safely omit the subscripts.

Money plays a special role in this scheme. We assume that all economic goods involved in exchange could be exchanged for money. So, if some of the members of an equivalence class $[g]$ are exchanged for goods, we can assign a monetary value to the barter transaction and use it in computing the average for the class.

As a standard of value, money - albeit time-varyingserves as a unit of measurement. Although not a perfect analogue of, say, the meter as a unit of length, money does function as a measure of market value. Note that a particular meter stick bears the same relationship to the meter (as a unit of length) as a particular dollar bill to the dollar (as a unit of exchange value). For example, the meter is infinitely divisible, but a meter stick is not. The same holds for the dollar in relation to the dollar bill. These two abstract units differ in that the meter is based on a constant feature of the physical universe, whereas the dollar (or other monetary unit) is based on properties of a social universe that is known to vary over time. ${ }^{9}$

Our formal definition of commodity is somewhat broader than the notions used intuitively by some economists and businesspeople. However, it reflects the actual state of affairs more accurately than these intuitive notions. In an advanced market economy, anything that can be appropriated and assigned a market value may be a commodity.

This observation is crucial for analyzing so-called information commodities because these kinds of commodities appear at first glance to be fundamentally

\footnotetext{
"The analogy between money and length applies only to their respective measurement properties. Money, unlike length, functions as a store of value and medium of exchange. It makes little sense to speak of storing length (as, for example, a collection of yard sticks), or to exchange a "store of length" for something else.
}

different from what we normally conceive to be commodities. With our definition, the differences dissolve.

\section{Information Commodities}

If information-whatever it may be-can be owned and valued, it can be a commodity. This gives us precise criteria for determining the commodity-hood of information. Can a piece of software be owned and priced? Appropriability and valuability are the key issues here, not the intangible character of an ided reflected in an algorithm or a computer program.

If property rights in a class of programs can be protected effectively, and if the programs can be exchanged for a nonzero amount of money or for commodities of nonzero value, then the class of programs may constitute a commodity. This line of reasoning lcads onc to ask two fundamental questions: (1) what precisely is to be protcctcd, and (2) how does software or any other putative information commodity acquire market value?

Lct us consider first what is needed to protect property rights in an arbitrary commodity. Then we will analyze the special case of property rights in information commodities.

According to our definition, each token of a commodity must have a unique owner. This implies means for keeping track of who owns what, and social mechanisms for protecting the interests of owners. Clearly, the idea of ownership is meaningless in the absence of a system of enforceable laws that defines property, and establishes procedures for determining and transferring ownership and settling disputes among claimants to property.

Protection of tangible or concrete commodities poses no major conceptual difficulty in the world of today. This is because tangible commodities like loaves of bread, cars, television sets, bushels of wheat, etc. are discrete physical objects whose use by one owner precludes use by any other owner. Once a loaf of bread has been consumed, it exists no more. The simplest form of ownership is socially sanctioned physical possession. Protection of ownership in this case requires reliable means for identifying owners, e.g., testimony of neighbors or officials, and insuring their ability to retain physical possession of their goods.

If tangible commodities can be held for their owners by third parties, identification of owners becomes more complicated. In this case, formal inventories must be maintained, and the inventories themselves protected against tampering. Thus, property rights in tangible commodities can be protected by a social system with: (1) reliable and secure inventories of ownership, and (2) effective means for securing persons and premises against theft of langible goods.

Abstract commodities share with their concrete counterparts the need for reliable inventory systems. Moreover, they must also be protected against theft. 
But theft may not involve unauthorized physical removal of property in the case of abstract commodities. Some abstract commodities (e.g., a financial instrument such as a bearer bond) have the same property characteristics as their concrete cousins (e.g., a television set). Ownership of bearer bonds requires essentially the same methods of protection as ownership of a television set. The differences between these two commodities have to do with the way they are used, not with protection of the property rights of their respective owners.

Nonnegotiable securities give greater importance to the third-party surrogate for the owner. The key to ownership in these cases is the record of ownership, a table showing the correspondence between a collection $\mathbf{S}$ of securities and a set of owners. (In terms of the notation introduced earlier, this record is just the restriction of the function $\mathbf{A}$ to the set $\mathbf{S}$.) Protection of property rights in this kind of commodity is tantamount to maintaining the security and integrity of the record system.

Thus far we have been speaking of conventional commodities, whether concrete (e.g., a loaf of bread) or abstract (e.g., registered securities). Are information commodities fundamentally different from the conventional ones? Certainly, information is different from energy and matter; but this does not necessarily imply that a commodity somehow made up of information inherits those differences.

Bread and information differ with respect to the results of consumption." When a loaf of bread is eaten, its essence is gone; but information is preserved under consumption: a book can be read and reread, a program executed rcpeatcdly, a database queried by many users, etc., with no degradation of the information contained in book, program, or database. What this suggests is that a given piece of information cannot be appropriated for exchange in the marketplace, because both seller and buyer have it after the former conveys it to the latter.

Nevertheless, books, computer programs, and databases are traded in the marketplace. Are these things economic anomalies? A new order of commodities? Not at all. The apparent contradiction disappears when one realizes that an information commodity is not the same thing as a piece of information. These commodities "contain" information in some sense, but they are commodities just like bread, television sets, and common stocks.

As argued above, anything that is appropriable and has market value may be turned into a commodity. Thus, information could be turned into a commodity if it is possible to incorporate it in something that can be appropriated and valued in the marketplace. In fact

\footnotetext{
${ }^{10}$ See Boulding (1966), Hall (1981), Braunstein (1981), and Cleveland (1982) for further discussion of the special economic characteristics of information.
}

business people have been doing just that with information for some considerable time. The market value of an information commodity derives from its capacity to support decision or control processes by furnishing information; but this capacity is only partly dependent on the specific information (ability to decide or control) furnished by the commodity.

An example, which purports to show how information functions in the marketplace, may help to clarify this distinction. Commodities traders are eager consumers of information released by the United States Department of Agriculture on the American wheat crop. Unexpected changes in estimated crop yields influence the price of wheat futures contracts-increases, unanticipated by market participants, tend to drive prices down, while decreases push them up. By selling short on reports of unexpected crop increases or buying long on reports of decreases, it is possible for traders to make handsome profits. Of course, to profit from such information, the trader would have to obtain and act on the reports before they become general knowledge, i.e., to trade on "inside information."

If it were possible to purchase the information (i.e., the ability to make the investment decision) in advance of the public announcement, how much should the trader be willing to pay for it? At first glance it seems reasonable to calculate its value in terms of the profit that could potentially be realized from acting on it as inside information. But how could this calculation be made in advance of having the information? ${ }^{11}$ There is no way to know whether to trade short or long, and no way to estimate the potential gains without having the information itself. So the trader cannot decide what to pay before having it; but if the seller were to allow the trader to see the report before being paid for the information, the seller might have a hard time collecting anything, regardless of the profit made by the trader. Assuming the trader is convinced that he or she will never again have occasion to purchase information from the informant, he or she may very well withhold payment on the grounds that nothing was actually taken from the informant.

As explained earlier, the information associated with a declarative statement that has no index other than itself has no exchange value. ${ }^{12}$ That is to say, an item whose usefulness cannot be assessed before being extracted in a way which precludes an exchange between a buyer and a seller is not a commodity. So, the unextractable information described in the example cannot be made into a commodity. The key to interpreting the example lies in the condition under which

\footnotetext{
${ }^{11}$ As Braunstein (1981, p. 11) puts it: "I cannot be certain of the value to me of a bit of information until I know what it is."

${ }^{12}$ Braunstein (1981, p. 11), elaborating on the remark cited earlier, observes: "In fact, I cannot make an accurate judgment on the basis of part of the information or on information about the information. And if I did have perfect information about what was being offered for sale, I would no longer need to purchase it."
} 
the trader is likely to withhold payment, i.e., when he has no intention of using the informant again. To determine in advance how much it is worth paying the informant, the trader must have reasonable expectations of gains to be made from information made available by this particular informant or by comparable ones under similar circumstances. That is to say, one must know enough to estimate the informant's ability to deliver information that will enable the trader to decide what commodity investments to make.

Thus we are led to define an information commodity as a commodity whose function it is to enable the user, a goal-seeking system, to obtain information, i.e., to obtain the ability to decide or control. Books, databases, computer programs, and advisory services are common examples of information commodities. Their market value derives from their capacity to furnish information..$^{13}$ Thus, we will examine the specific contributions of various attributes of information commodities to their capacity to inform, and hence their respective contributions to market value.

The definition of information commodity given here is designed to permit systematic analysis of the elements that give such commodities value in the marketplace. Every information commodity has a kernel of information, which is the particular "ability to decide or control" sought by a potential user. However, that kernel must be carried by an agent, whether a human being or an artifact, and must be accessible to a potential user in appropriate form to be of value. ${ }^{14}$ Information-other than the kernel-may again come into play here, but so do other ingredients. These we will explain in the sequel (Mowshowitz, 1991a) to this article.

\section{Acknowledgments}

This research was supported by the National Science Foundation, Division of Information, Robotics, and Intelligent Systems under grant number IRI-8800441.

The author is indebted to Professor Yale Braunstein, University of California, Berkeley, for his help in navigating through the literature of information economics, and for his review of the report on which this article is based. The author is also indebted to Professors Giuseppe Trautteur and Giovanni Criscuolo, University of Naples, for their critical reading of the report; and to Professor Claude Faucheux, Erasmus UniversityRotterdam, for his comments on early drafts of the report. Of course, the author accepts full responsibility for the ideas presented here.

\footnotetext{
${ }^{13}$ Compare with Rouse's (1986) discussion of the value of information in a decision-support system.

${ }^{14}$ Taylor (1982a, p. 342) distinguishes between the "content of the message" and the "service that provides the message;" or (Taylor, 1982b, p. 310) between content and "the message packages and the technology and systems used to generate, store, organize, present, and move these packages."
}

\section{References}

Arrow, K. J. (1979). The economics of information. In M. Dertouzos \& J. Moses J. (Eds.), The computer age (pp. 306-317). Cambridge, MA: MIT Press.

Boulding, K.E. (1966). The economics of knowledge and the knowledge of economics. American Economic Review, 56, 1-13.

Braunstein, Y. M. (1981). Information as a commodity: public policy issues and recent research. In R.M. Mason \& J. E. Creps (Eds.), Information services: Economics, management, and technology (pp. 9-22). Boulder, CO: Westvicw Press.

Cleveland, H. (1982). Information as a resource. The Futurist, 3-4, $34-39$.

Hall, K. (1981). The economic nature of information. The Information Society, 1, 143-166.

Hintikka, J. (1984). Some varieties of information. Information Processing \& Management, 20, 175-181.

Hirshleifer, J. (1973). Where are we in the theory of information? American Economic Review, 63, 31-39.

Hoffmann, E. (1980). Defining information: an analysis of the information content of documents. Information Processing \& Management, 16, 291-304.

King, D.W. (1982). Marketing secondary information products and services. Journal of the American Society for Information Science, $32,168-174$

Lamberton, D. M. (1984a). The emergence of information economics. In M. Jussawalla \& H. Ebenfield (Eds.), Communication and information economics: New perspectives (pp. 7-22). Amsterdam: North-Holland.

Lamberton, D. M. (1984h). The economics of information and organization. In M. E. Williams (Ed.), Annual review of information science and technology, volume 19 (pp. 3-30). White Plains, N Y: Knowledge Industry Publications.

Langlois, R. N. (1982). Systems theory and the meaning of information. Journal of the American Society for Information Science, 32, 395-399.

Marschak, J. (1959). Remarks on the economics of information. In Contributions to Scientific Research in Management. Proceedings of the Scientific Program following the Dedication of the Western Data Processing Center. Graduate School of Business Administration, University of California, Los Angeles.

Mowshowitz, A. (1987). The information marketplace. Background Report, Office of Technology Assessment, U.S. Congress, Washington, DC.

Mowshowitz, A. (1992a). On the market value of information commodities: II. Supply price. Journal of the American Society for Information Science, 43, 233-241.

Mowshowitz, A. (1992b). On the market value of information commodities: III. Demand price. Journal of the American Society for Information Science, 43, 242-248.

Rouse, W. B. (1986). On the value of information in system design: a framework for understanding and aiding designers. Information Processing \& Management, 22, 217-228.

Shannon, C. E. (1948 July/October). The mathematical theory of communication. Bell System Technical Journal. Reprinted in Shannon, C. E. \& Weaver, W. (1959). The Mathematical Theory of Communication. Urbana, IL: University of Illinois Press.

Taylor, R.S. (1982a). Value-added processes in the information life cycle. Journal of the American Society for Information Science, 32 , 341-346.

Taylor, R. S. (1982b). Organizational information environments. In G. P. Sweeny (Ed.), Information and the transformation of society (pp. 309-322). Amsterdam: North-Holland.

Winograd, T. (1975). Frame representation and the declarative/ procedural controversy. In D. G. Bobrow \& A. Collins (Eds.), Representation and understanding: Studies in cognitive science (pp. 185-210). New York: Academic Press. 